\title{
Measurement of CD74 N-terminal Fragment Accumulation in Cells Treated with SPPL2a Inhibitor
}

Rubén Martínez-Barricarte ${ }^{1, *}$, Xiao-Fei Kong ${ }^{1}$ and Jean-Laurent Casanova ${ }^{1,2,3,4,5}$

${ }^{1}$ St. Giles Laboratory of Human Genetics of Infectious Diseases, Rockefeller Branch, The Rockefeller University, New York City, NY, USA; ${ }^{2}$ Laboratory of Human Genetics of Infectious Diseases, Necker Branch, INSERM U1163, Necker Hospital for Sick Children, Paris, France; 'PParis Descartes University, Imagine Institute, Paris, France; ${ }^{4}$ Howard Hughes Medical Institute, New York, USA; ${ }^{5}$ Pediatric Hematology and Immunology Unit, Necker Hospital for Sick Children, AP-HP, Paris, France *For correspondence: $\underline{\text { rmartinezb@rockefeller.edu }}$

[Abstract] The recent discovery of human signal peptide peptidase-like 2a (SPPL2a) deficiency in humans revealed the toxicity associated with the accumulation of one of its substrates, CD74 N-terminal fragment (CD74-NTF), for certain type of dendritic cells (CDC2). We developed a two-step protocol for monitoring the accumulation of this molecule in different subsets of PBMCs and immortalized B cells, in which SPPL2a is chemically inhibited and CD74-NTF levels are then assessed by flow cytometry or western blotting. The chemical inhibition of SPPL2a has been described elsewhere, but this is the first time that this inhibition has been reported as a protocol.

Keywords: SPPL2a, CD74-NTF, Inhibition, Chemical, L-685,458

[Background] Signal peptide peptidase-like 2A (SPPL2a) is a transmembrane intracellular protease from the GxGD family (Voss et al., 2013). In both humans and mice, the absence of this enzyme results in the accumulation of one of its substrates, CD74 N-terminal fragment (CD74-NTF) in MHC class IIpositive cells (Beisner et al., 2013; Bergmann et al., 2013; Schneppenheim et al., 2013; Kong et al., 2018). This accumulated CD74-NTF is selectively toxic to B cells in mice (Beisner et al., 2013; Bergmann et al., 2013; Schneppenheim et al., 2013) and conventional dendritic cells type 2 (cDC2) in both humans $\left(C D 1 c^{+} D C s\right)$ and mice $\left(C D 11 c^{+} C D 11 c^{+} D C s\right)$. We identified the cells most sensitive to CD74-NTF accumulation and gained insight into the mechanism of CD74-NTF-mediated cytotoxicity, using a specific chemical inhibitor of SPPL2a (L-685,458) (Huttl et al., 2015) in peripheral blood mononuclear cells (PBMCs) and Epstein Barr virus-immortalized B cells (EBV-B) from healthy controls. We analyzed CD74-NTF accumulation by fluorescence-activated cell sorting (FACS) or western blotting (WB).

\section{Materials and Reagents}

1. $1.5 \mathrm{ml}$ microcentrifuge tube (Fisher Scientific, catalog number: 21-402-905)

2. 24-well plate (Stemcell, catalog number: 38021)

3. Plate with 96 V-bottomed wells (Denville Scientific, catalog number: B1201-S)

4. EBV-B cells were produced in-house, as previously described (Hui-Yuen et al., 2011) 
5. PBMCs were isolated from fresh blood, as previously described (Panda et al., 2012; Panda and Ravindran, 2013)

6. Tris (MP Biomedicals, catalog number: 103133)

7. Tween 20 (Fisher BioReagents, catalog number: BP337-500)

8. WB stripping buffer (Thermo Fisher Scientific, catalog number: PI-21063)

9. $20 \%$ SDS (VWR Life Sciences, catalog number: 0837-200ML)

10. 4-12\% Criterion ${ }^{\mathrm{TM}}$, XT Bis-Tris gel (Bio-Rad, catalog number: 345-0124)

11. $5 \%$ non-fat milk powder (Bio-Rad, catalog number: $170-6404$ )

12. Anti-CD11c-PerCP-Cy5.5 antibody (BioLegend, BU15, catalog number: 307624)

13. Anti-CD123-PE-Cy7 antibody (BioLegend, 6H6, catalog number: 30601)

14. Anti-CD141-PE antibody (BioLegend, M80, catalog number: 344104)

15. Anti-CD1C-APC-Cy7 antibody (BioLegend, L161, catalog number: 331520)

16. Anti-CD20-PE antibody (BD Biosciences, $2 \mathrm{H} 7$, catalog number: 555623 )

17. Anti-CD56-Alexa700 antibody (BD Biosciences, B159, catalog number: 557919)

18. Anti-CD74 antibody (Abcam, catalog number: ab97479)

19. Anti-GAPDH HRP-conjugated antibody (Santa Cruz, catalog number: sc47724 HRP)

20. Anti-HLA-DR-V450 antibody (BD Biosciences, L243, catalog number: 642285)

21. Anti-mouse-Alexa 488 secondary antibody (Life Technologies, catalog number: A-21131)

22. Anti-rabbit HRP-conjugated secondary antibody (GE Healthcare Life Sciences, catalog number: NA934V)

23. BD Cytofix/Cytoperm (BD, catalog number: 554722)

24. BioRad protein assay dye reagent concentrate (BioRad, catalog number: 5000006)

25. Bovine serum albumin (BSA) (Calbiochem or Millipore)

26. Bromphenol blue (Sigma-Aldrich, catalog number: B75808-5G)

27. CD14 microbeads (Miltenyi Biotec, catalog number: 130-050-201)

28. CD19 microbeads (Miltenyi Biotec, catalog number: 130-050-301)

29. CD3 microbeads (Miltenyi Biotec, catalog number: 130-050-101)

30. CD56 microbeads (Miltenyi Biotec, catalog number: 130-050-401)

31. cOmplete protease inhibitor cocktail (Roche, catalog number: 11873580001)

32. DAPT gamma secretase inhibitor (R\&D Systems, 2634/10)

33. DMSO (MP Biomedicals, catalog number: 196055)

34. 0.5 M EDTA (Fisher Scientific, catalog number: BP2482-1)

35. Ethanol 200 Proof (Decon Laboratories, catalog number 2716)

36. Full-Range Rainbow Molecular Weight Marker (GE Healthcare Life Sciences, catalog number: RPN88E)

37. Glycerol (Fisher Chemical, catalog number: G33-500)

38. Glycine (Fisher BioReagents, catalog number: BP381-1)

39. Heat-inactivated fetal calf serum (FCS) (Thermo Fisher Scientific, catalog number: A3382001)

40. L-685,458 (EMD Millipore, catalog number: 565771) 
41. Live/DEAD Fixable Aqua (Life Technologies, catalog number: L34957)

42. $\mathrm{NaCl}$ (Fisher Scientific, catalog number: S271-3)

43. NP40 (Sigma-Aldrich, catalog number: I8896-100ML)

44. NUPAGETM MES SDS Running Buffer (20x) (Thermo Fisher Scientific, catalog number: NP0002)

45. PBS (Mediatech, catalog number: 21-0310 CVR)

46. Pierce ECL substrate (Thermo Fisher Scientific, catalog number: 32106)

47. PVDF membrane (Millipore, catalog number: IPVH00010)

48. RPMI medium (Life Technologies, catalog number: 61870-127)

49. Saponin (Sigma-Aldrich, catalog number: 47036-50G-F)

50. Sodium deoxycholate (Sigma-Aldrich, catalog number: D6750-100G)

51. Tris- $\mathrm{HCl}$

52. Culture media (see Recipes)

53. RIPA buffer (see Recipes)

54. 5x Laemmli loading buffer (see Recipes)

55. TBS-T (see Recipes)

56. Blocking buffer (see Recipes)

57. FACS buffer (see Recipes)

58. Intracellular staining buffer (see Recipes)

59. Transfer buffer (see Recipes)

\section{Equipment}

1. Amersham Imager 600 (GE Healthcare, catalog number: 29083461)

2. BD LSR-II (BD Biosciences) with BDFACSDIVA software (BD Biosciences) and FlowJo software for data analysis

3. Centrifuge $5418 \mathrm{R}$ (Eppendorf, catalog number: 022620304)

4. Criterion Vertical Electrophoresis cells (Bio-Rad, catalog number: 1656001)

5. Eppendorf centrifuge 5810R (Eppendorf, catalog number: 022625004)

6. Fisher Scientific Vortex Mixer (Fisher Scientific, catalog number: 02215365)

7. Forma ${ }^{\mathrm{TM}}$ Series 3 Water Jacketed $\mathrm{CO}_{2}$ Incubator (Thermo Fisher, catalog number: 4110)

8. Labnet Rocking Platform Shaker, Single, 230 VAC (Labnet, catalog number UX-51401-07)

9. Pipette 4-pack $(2.5,10,100$ and 1,000 $\mu$ l volumes) and pipette tips (Eppendorf, catalog number: 05-403-151)

10. PowerPac ${ }^{\mathrm{TM}} \mathrm{HC}$ High-Current Power Supply (BioRad, catalog number: 1645052)

11. Trans-Blot ${ }^{\circledR}$ SD Semi-Dry Transfer Cell (BioRad, catalog number: 1703940) 


\section{Procedure}

A. Chemical inhibition of SPPL2a in PBMCs and EBV-B cells

1. Plate $10^{6}$ EBV-B cells or PBMCs (both grown in suspension) in triplicate, in culture medium, at a density of $10^{6} \mathrm{cells} / \mathrm{ml}$ in a 24 -well plate.

2. Immediately after plating, treat one well with $1 \mu \mathrm{M}$ of DMSO, a second well with $10 \mu \mathrm{M}$ DAPT (generic gamma secretase inhibitor used as a negative control because it does not inhibit SPPL2a; on delivery, it should be dissolved in DMSO to yield a $100 \mathrm{mM}$ solution, which is then stored at $-20^{\circ} \mathrm{C}$, protected against freeze-thaw cycles cycles) and a third one should be treated with a $1 \mu \mathrm{M}$ solution of the gamma secretase/SPPL inhibitor L-685,458 (stock solution at $1 \mathrm{mM}$, storage at $-20^{\circ} \mathrm{C}$, protected against freeze-thaw cycles).

3. Incubate the cells at $37{ }^{\circ} \mathrm{C}$, under an atmosphere containing $5 \% \mathrm{CO}_{2}$, in a tissue culture incubator for $24 \mathrm{~h}$, without shaking.

B. Western-blot analysis of CD74-NTF accumulation in EBV-B cells

1. After treatment, transfer the EBV-B cells into a $1.5 \mathrm{ml}$ microcentrifuge tube and centrifuge them for $5 \mathrm{~min}$ at $300 \times \mathrm{g}$ at room temperature.

2. Remove the supernatant.

3. Resuspend the EBV-B cells in $2 \mathrm{ml}$ of PBS and centrifuge them again for $5 \mathrm{~min}$ at $300 \times \mathrm{g}$ at room temperature.

4. Remove the supernatant.

5. Resuspend the EBV-B cells in $30 \mu \mathrm{l}$ of ice-cold RIPA buffer.

6. Vortex for $30 \mathrm{~s}$.

7. Incubate on ice for $20 \mathrm{~min}$.

8. Centrifuge at $18,000 \times \mathrm{g}$ for $20 \mathrm{~min}$ at $4{ }^{\circ} \mathrm{C}$.

9. Collect the supernatant containing the protein.

10. Assess protein concentration with the BioRad protein assay according to the kit manufacturer's instructions (BioRad protein assay).

11. Mix $50 \mu \mathrm{g}$ of protein with a $1 / 5$ volume of $5 x$ Laemmli loading buffer.

12. Incubate for $5 \mathrm{~min}$ at $55^{\circ} \mathrm{C}$.

13. Perform electrophoresis in a precast 4-12\% acrylamide SDS-PAGE gel in MES buffer, with $5 \mu \mathrm{l}$ of Full-Range Rainbow Molecular Weight Marker as the size standard, according to the manufacturer's instructions (A Guide to Polyacrylamide Gel Electrophoresis and Detection).

14. Transfer proteins to a PVDF membrane according to the manufacturer's instructions (Immobilon-P-PVDF membrane).

15. Block the membrane by incubating for $1 \mathrm{~h}$ at room temperature in blocking buffer.

16. Remove the blocking buffer and wash the membrane with TBS-T. 
17. Incubate the membrane overnight, with shaking, with an anti-CD74 antibody specifically recognizing the NTF of CD74 (cleaved or uncleaved) diluted 1:1,000 in 1\% BSA in TBS-T at $4{ }^{\circ} \mathrm{C}$.

18. The following day, wash the membrane four times by incubation with TBS-T, for 5 min per wash, on an orbital shaker, at room temperature.

19. Add anti-rabbit HRP-conjugated secondary antibody to the membrane at a dilution of 1:5,000 in $1 \%$ BSA in TBS-T and incubate for $30 \mathrm{~min}$ on an orbital shaker at room temperature.

20. Wash the membrane five times, as described above (point 18), at room temperature.

21. Develop the membrane with ECL substrate according to the manufacturer's instructions (Pierce ${ }^{\mathrm{TM}}$ ECL Western Blotting Substrate). The signal should become visible after exposure for five minutes.

22. Strip the membrane with WB stripping buffer at room temperature.

23. Block the membrane by incubation for $1 \mathrm{~h}$ in blocking buffer at room temperature.

24. Remove the blocking buffer and wash the membrane with TBS-T at room temperature.

25. Incubate the membrane for $1 \mathrm{~h}$, with shaking, with HRP-conjugated anti-GAPDH antibody diluted 1:5,000 in 1\% BSA in TBS-T at room temperature.

26. Wash the membrane five times by incubating with TBS-T, for five minutes per wash, on an orbital shaker at room temperature.

27. Develop the membrane with ECL substrate according to the manufacturer's instructions (Pierce ${ }^{\mathrm{TM}} \mathrm{ECL}$ Western Blotting Substrate). The signal should become visible after 2 min of exposure.

C. FACS analysis of CD74-NTF accumulation in different PBMC populations

1. Centrifuge PBMCs at $300 \times g$ for 5 min.

2. Remove supernatant and resuspend PBMCs in FACS buffer and centrifuge again at $300 \times g$ for 5 min.

3. Isolate $\mathrm{CD} 14^{+}$cells by MACS (CD14 microbeads) according to the manufacturer's instructions (CD14 MicroBeads, human).

4. Isolate $\mathrm{CD}^{+}$cells from the flow through ( $\mathrm{CD} 14^{-}$), by MACS (CD3 microbeads) according to the manufacturer's instructions (CD3 microbeads, human).

5. Isolate $\mathrm{CD} 19^{+}$and $\mathrm{CD} 56^{+}$cells from the flow through (CD14, CD3-) by MACS (CD19 and CD56 microbeads) according to the manufacturer's instructions (CD19 Microbeads, human and CD56 microbeads, human).

6. Wash these four fractions-CD14 ${ }^{+}, \mathrm{CD}^{+}, \mathrm{CD} 19^{+} \mathrm{CD} 56^{+}$cells and the flow through (CD14-, CD3-, CD19-, CD56-)-twice by resuspension in $2 \mathrm{ml}$ of FACS buffer and centrifugation at $300 \times \mathrm{g}$ for 5 min, removing the supernatant.

7. Stain the cells in a 96-well plate with V-bottomed wells. Re-suspend fractions 1 and 2 (CD14 ${ }^{+}$ and $\left.\mathrm{CD}^{+}\right)$in $50 \mu \mathrm{l}$ of FACS buffer with Live/Dead Fixable Aqua 1:1,000. Re-suspend fractions $3\left(\mathrm{CD} 19^{+} \mathrm{CD} 56^{+}\right)$in $50 \mu \mathrm{l}$ of FACS buffer with Live/Dead Fixable Aqua 1:1,000, anti-CD20-PE 
and anti CD56-Alexa700 antibodies. Re-suspend fractions 4 (CD14, CD3-, CD19-, CD56-) in 50 $\mu \mathrm{l}$ of FACS buffer with Live/Dead Fixable Aqua 1:1,000, anti-CD11c-PerCP-Cy5.5, anti-HLADR-V450, anti-CD123-PE-Cy7, anti-CD1C-APC-Cy7 and anti-CD141-PE antibodies (Table 1). Use all antibodies at a dilution of 1:50, and incubate the cells with the corresponding mixture for 30 min on ice.

Table 1. Staining panels

\begin{tabular}{|l|l|l|}
\hline Fraction & Populations contained & Antibodies and dyes \\
\hline 1 & CD14 $^{+}$ & LDA \\
\hline 2 & CD3 $^{+}$ & LDA \\
\hline \multirow{3}{*}{3} & \multirow{3}{*}{ CD19 $^{+}$CD56 $^{+}$} & LDA \\
\cline { 3 - 3 } & & Anti-CD20-PE \\
\cline { 3 - 3 } & & Anti-CD56-Alexa700 \\
\hline \multirow{4}{*}{4} & \multirow{3}{*}{ CD14-CD3-CD19-CD56- } & LDA \\
\cline { 3 - 3 } & & Anti-CD11C-PerCP-Cy5.5 \\
\cline { 3 - 3 } & & Anti-HLA-DR-V450 \\
\cline { 3 - 3 } & & Anti-CD123-PE-Cy7 \\
\cline { 3 - 3 } & & Anti-CD1C-APC-Cy7 \\
\cline { 3 - 3 } & & Anti-CD141-PE \\
\hline
\end{tabular}

LDA: Live/Dead Fixable Aqua

8. Wash cells twice by adding $100 \mu$ l of FACS buffer and centrifuging the plate at $350 \times g$ for $3 \mathrm{~min}$.

9. Fix and permeabilize the cells with $100 \mu \mathrm{l}$ of BD Cytofix/Cytoperm according to the manufacturer's instructions (Fixation and Permeabilization Solution).

10. Perform intracellular staining by incubation with $50 \mu \mathrm{l}$ of anti-CD74 antibody diluted 1:500 in intracellular staining buffer for $45 \mathrm{~min}$ at $4{ }^{\circ} \mathrm{C}$.

11. Wash cells twice each with $100 \mu$ of intracellular staining buffer.

12. For the final staining step, resuspend cells in $50 \mu \mathrm{l}$ of intracellular staining buffer with antimouse-Alexa 488 secondary antibody diluted 1:1,000.

13. Finally, wash the cells twice with FACS buffer and resuspend them in $100 \mu$ l of FACS buffer.

14. Collect data in an LSR-II at a rate of 1000 events per second with FACS diva software, after appropriate compensation.

\section{Data analysis}

1. On western blots, the accumulation of CD74-NTF can be assessed by monitoring the appearance of a $9 \mathrm{kDa}$ band exclusively in the samples treated with L-685,458. (Figure 2E in https://www.ncbi.nlm.nih.gov/pmc/articles/PMC6130844/). 
2. Lymphocytes and monocytes are gated individually on the basis of their vital status (alive, LDA) side-scattering (SSC) and forward-scattering (FSC) properties. $\mathrm{CD}^{+}$cells are considered to correspond to $\mathrm{T}$ cells, CD20+ cells to $\mathrm{B}$ cells, $\mathrm{CD} 56^{+}$to NK cells and $\mathrm{CD} 14^{+}$to monocytes. For DC gating, pDCs are considered to be $\mathrm{HLA}-\mathrm{DR}{ }^{+} \mathrm{CD} 11 \mathrm{c}^{-} \mathrm{CD} 123^{+}, \mathrm{CDC1}$ are HLA$\mathrm{DR}^{+} \mathrm{CD} 11 \mathrm{c}^{+} \mathrm{CD} 11 \mathrm{c}^{+} \mathrm{CD} 141^{+}$and $\mathrm{CDC} 2$ are $\mathrm{HLA}-\mathrm{DR}{ }^{+} \mathrm{CD} 11 \mathrm{c}^{+} \mathrm{CD} 1 \mathrm{c}^{+}$. After gating, the MFI for CD74 is assessed with FlowJo software, using the values for untreated cells and cells treated with DAPT as negative controls. A detail gating strategy and the expected results are presented in our original paper (Figures $2 \mathrm{H}$ and $\mathrm{S} 2$ in https://www.ncbi.nlm.nih.gov/pmc/articles/PMC6130844/).

\section{Notes}

1. We suggest performing this protocol in triplicate for each set of conditions, to obtain reproducible, reliable and robust results.

2. Given that the expected size of the protein of interest is very small $(9 \mathrm{kDa})$, check the protein weight marker carefully to ensure that the CD74-NTF does not run off the end of the gel.

3. The choice of the FACS staining strategy described here was based on our reagent/equipment availability and the planned follow-up experiments to reduce variability. However, any other custom staining strategy may be used, according to individual needs. In addition, any other CD74/SPPL2a-expressing cell line or cell type can be studied with this protocol.

4. We analyzed CD74-NTF accumulation in EBV-B cells by western blotting because this approach is more visual and we had unlimited numbers of these cells available. We analyzed T, B, NK, DC and monocytes by FACS due to the limited availability of these cells. However, if sufficient numbers of PBMCs can be obtained, the individual cell populations can be purified and CD74NTF accumulation can be analyzed by western blotting. Conversely, CD74-NTF accumulation can also be studied by FACS.

5. For any doubts or questions, contact the first author at rmartinezb@rockefeller.edu.

\section{$\underline{\text { Recipes }}$}

1. Culture media

RPMI medium (Life Technologies) supplemented with 10\% heat-inactivated fetal calf serum (FCS) (Thermo Fisher Scientific)

2. RIPA buffer

$50 \mathrm{mM}$ Tris $\mathrm{pH} 8$

1 mM EDTA

$150 \mathrm{mM} \mathrm{NaCl}$

$0.5 \%$ sodium deoxycholate

$1 \%$ NP40 
0.1\% SDS and cOmplete protease inhibitor cocktail (Roche)

3. $5 x$ Laemmli loading buffer

$80 \mathrm{mM}$ Tris- $\mathrm{HCl} \mathrm{pH} 6.8$

$2 \%$ SDS

$10 \%$ glycerol

$0.02 \%$ bromphenol blue

4. TBS-T

$20 \mathrm{mM}$ Tris $\mathrm{pH} 7.4$

$150 \mathrm{mM} \mathrm{NaCl}$

$0.1 \%$ Tween 20

5. Blocking buffer

TBS-T 5\% non-fat milk powder and 2.5\% BSA

6. FACS buffer

2\% FCS in PBS

7. Intracellular staining buffer

PBS

$5 \%$ BSA

$0.1 \%$ saponin

8. Transfer buffer

$192 \mathrm{mM}$ glycine

$25 \mathrm{mM}$ Tris

$20 \%$ ethanol

\section{Acknowledgments}

We thank all the authors of our original research article (Kong et al., 2018). R.M.B. was funded by a European Molecular Biology Organization (EMBO) long-term fellowship. X.-F.K. was supported by the Jerome Lejeune Foundation, the Stony Wold-Herbert Fund, the Choh-Hao Li Memorial Fund Scholar Award, and the Shanghai Educational Development Foundation. The Laboratory of Human Genetics of Infectious Diseases was supported by grants from the St. Giles Foundation (J.-L.C.), The Rockefeller University Center for Clinical and Translational Science (grant UL1TR001866 from the National Center for Research Resources and the National Center for Advancing Sciences (NCATS) to R.M.-B. and X.-F.K.), the National Institutes of Health, the National Institute of Allergy and Infectious Diseases (5R01AI089970-02 and 5R37AI095983 to J.-L.C.), Institut National de la Santé et de la Recherche Médicale (INSERM), Paris Descartes University, and The Rockefeller University. 


\section{Competing interests}

The authors have no competing interests to declare.

\section{Ethics}

This study was approved by and performed in accordance with the requirements of the institutional ethics committee of The Rockefeller University Hospital, New York, USA. Informed consent was obtained for all healthy control volunteers participating in the development of this protocol.

\section{$\underline{\text { References }}$}

1. Beisner, D. R., Langerak, P., Parker, A. E., Dahlberg, C., Otero, F. J., Sutton, S. E., Poirot, L., Barnes, W., Young, M. A., Niessen, S., Wiltshire, T., Bodendorf, U., Martoglio, B., Cravatt, B. and Cooke, M. P. (2013). The intramembrane protease Sppl2a is required for B cell and DC development and survival via cleavage of the invariant chain. J Exp Med 210(1): 23-30.

2. Bergmann, H., Yabas, M., Short, A., Miosge, L., Barthel, N., Teh, C. E., Roots, C. M., Bull, K. R., Jeelall, Y., Horikawa, K., Whittle, B., Balakishnan, B., Sjollema, G., Bertram, E. M., Mackay, F., Rimmer, A. J., Cornall, R. J., Field, M. A., Andrews, T. D., Goodnow, C. C. and Enders, A. (2013). B cell survival, surface BCR and BAFFR expression, CD74 metabolism, and CD8dendritic cells require the intramembrane endopeptidase SPPL2A. J Exp Med 210(1): 31-40.

3. Huttl, S., Klasener, K., Schweizer, M., Schneppenheim, J., Oberg, H. H., Kabelitz, D., Reth, M., Saftig, P. and Schroder, B. (2015). Processing of CD74 by the intramembrane protease SPPL2a is critical for B cell receptor signaling in transitional B cells. J Immunol 195(4): 1548-1563.

4. Hui-Yuen, J., McAllister, S., Koganti, S., Hill, E. and Bhaduri-McIntosh, S. (2011). Establishment of Epstein-Barr virus growth-transformed lymphoblastoid cell lines. J Vis Exp(57).

5. Kong, X. F., Martinez-Barricarte, R., Kennedy, J., Mele, F., Lazarov, T., Deenick, E. K., Ma, C. S., Breton, G., Lucero, K. B., Langlais, D., Bousfiha, A., Aytekin, C., Markle, J., Trouillet, C., Jabot-Hanin, F., Arlehamn, C. S. L., Rao, G., Picard, C., Lasseau, T., Latorre, D., Hambleton, S., Deswarte, C., Itan, Y., Abarca, K., Moraes-Vasconcelos, D., Ailal, F., Ikinciogullari, A., Dogu, F., Benhsaien, I., Sette, A., Abel, L., Boisson-Dupuis, S., Schroder, B., Nussenzweig, M. C., Liu, K., Geissmann, F., Tangye, S. G., Gros, P., Sallusto, F., Bustamante, J. and Casanova, J. L. (2018). Disruption of an antimycobacterial circuit between dendritic and helper T cells in human SPPL2a deficiency. Nat Immunol 19(9): 973-985.

6. Schneppenheim, J., Dressel, R., Huttl, S., Lullmann-Rauch, R., Engelke, M., Dittmann, K., Wienands, J., Eskelinen, E. L., Hermans-Borgmeyer, I., Fluhrer, R., Saftig, P. and Schroder, B. (2013). The intramembrane protease SPPL2a promotes B cell development and controls endosomal traffic by cleavage of the invariant chain. J Exp Med 210(1): 41-58.

7. Panda, S. K. and Ravindran, B. (2013). Isolation of human PBMCs. Bio-protocol 3(3): e323. 
8. Panda, S. K., Kumar, S., Tupperwar, N. C., Vaidya, T., George, A., Rath, S., Bal, V. and Ravindran, B. (2012). Chitohexaose activates macrophages by alternate pathway through TLR4 and blocks endotoxemia. PLoS Pathog 8(5): e1002717.

9. Voss, M., Schroder, B. and Fluhrer, R. (2013). Mechanism, specificity, and physiology of signal peptide peptidase (SPP) and SPP-like proteases. Biochim Biophys Acta 1828(12): 2828-2839. 